\title{
Fréchet Derivatives of the Power Function
}

\author{
RAJENDRA BHATIA \& JOHN A. HOLBROOK
}

ABSTRACT. Let $A \rightarrow A^{r}$ be the map that takes a positive definite matrix to its $r$ th power, and let $D A^{r}$ be the Fréchet derivative of this map. It is known that $\left\|D A^{r}\right\|=\left\|r A^{r-1}\right\|$ if $r \leq 1$ or if $r \geq 2$, and also that this is not so if $1<r<\sqrt{2}$. We show that this equality between norms is true also when $\sqrt{2} \leq r<2$, and thus resolve this question completely. Motivation for such results comes from the need for perturbation bounds on the power functions; we discuss this application.

\section{INTRODUCTION}

For each real number $r$, consider the map on $n \times n$ positive (definite) matrices taking $A$ to $A^{r}$. This map is Fréchet differentiable and its derivative at $A$ is a linear map on the space of Hermitian matrices. This linear map $D A^{r}$ is defined as

$$
D A^{r}(B)=\lim _{t \rightarrow 0} \frac{(A+t B)^{r}-A^{r}}{t} .
$$

Several recent papers have examined the norm of this linear map.

If $B$ commutes with $A$, then it is clear (by simultaneously diagonalizing $A$ and $B)$ that $D A^{r}(B)=r A^{r-1} B$. So, if we restrict ourselves to the space of matrices that commute with $A$, we have

$$
\left\|D A^{r}\right\|=\left\|r A^{r-1}\right\| .
$$

It is remarkable that this norm relation remains true in the noncommutative case for several values of $r$. It is even more remarkable that this is true for all values of 
$r$ except those in the interval $(1, \sqrt{2})$. Completing the proof of this statement is the major outcome of this paper.

It was observed in [2] that $\left\|D A^{r}\right\|=\left\|r A^{r-1}\right\|$ holds when $0 \leq r \leq 1$, and when $r$ is a positive integer. Bhatia and Sinha [7] then showed that this is true for all $r<0$ and for all $r \geq 2$. Moreover, for $1<r<\sqrt{2}$ they constructed examples of $2 \times 2$ matrices $A>0$ and $B=B^{*}$ such that $\left\|D A^{r}(B)\right\|>r\left\|A^{r-1}\right\| \cdot\|B\|$. The question was left unresolved for $\sqrt{2} \leq r<2$. The main result in this paper plugs this gap:

Theorem 1.1. For any positive definite matrix $A$ and for $\sqrt{2} \leq r<2$, we have

$$
\left\|D A^{r}\right\|=\left\|r A^{r-1}\right\| .
$$

Our methods for evaluating $\left\|D A^{r}\right\|$ are quite different from the ones used in [7] and in the subsequent [8]. The first of these papers relied on integral representations of the functions $f(t)=t^{r}$ and the second on power series. We, on the other hand, compute the Schur multiplier norm of certain matrices associated with the Fréchet derivative. This method may be found useful in other calculations involving noncommutative derivatives. However, at present our results are limited to matrices, while the earlier results for other values of $r$ applied to infinite-dimensional operators as well.

Choose an orthonormal basis in which $A$ is diagonal: $A=\operatorname{diag}\left(p_{1}, \ldots, p_{n}\right)$. Then the linear operator $D A^{r}$ acts as Schur multiplication (entrywise multiplication) by the divided difference matrix

$$
\left[\frac{p_{i}^{r}-p_{j}^{r}}{p_{i}-p_{j}}\right]
$$

See [4], p. 124. For convenience, we assume that $1=p_{1}>p_{2}>\cdots>0$, and let $S_{r}$ be the matrix defined by

$$
r S_{r}=\left[\frac{p_{i}^{r}-p_{j}^{r}}{p_{i}-p_{j}}\right] .
$$

Then, the equality (2) says that

$$
\left\|S_{r}\right\|_{S H}=1
$$

where, for any matrix $M,\|M\|_{S H}$ denotes the Schur-Hermite norm of $M$, i. e., its norm as a Schur multiplier $B \rightarrow M \circ B$ on the space of Hermitian matrices. This norm is no greater than $\|M\|_{S}$, the Schur multiplier norm of $M$ on the space of all matrices. Since the $(1,1)$ entry of $S_{r}$ is $1,\left\|S_{r}\right\|_{S H} \geq 1$. So, to prove (2) it suffices 
to show that $\left\|S_{r}\right\|_{S} \leq 1$. By a theorem of Haagerup (see [12], [1], or [10], for example) this is so if and only if there is a factorization

$$
S_{r}=R C,
$$

where the rows of $R$ and the columns of $C$ are of norm at most 1. Our proof of Theorem 1.1 depends on showing the existence of such a factorization for the given values of $r$. We note that we actually have the equality $\|M\|_{S H}=\|M\|_{S}$ whenever $M$ is Hermitian (a result proved earlier by Mathias [15]), although we only need here the obvious inequality.

The special, and somewhat mysterious, role the number $\sqrt{2}$ plays is due to the following proposition.

Proposition 1.2. Let $1<r<2$. Then the inequality

$$
\left(\frac{1-p^{r}}{r(1-p)}\right)^{2} \leq \frac{1+p^{r-1}}{2}
$$

holds for all $p \in(0,1)$ if and only if $r \geq \sqrt{2}$.

We need this proposition to demonstrate the Haagerup factorization mentioned above. While for $r \geq 3 / 2$ it is easy to prove the inequality (4) by simple convexity arguments, we are forced to use rather intricate and unusual arguments to prove it for all $r \geq \sqrt{2}$.

The proof of the main result is given in Section 2. In Section 3 we reinterpret the results in terms of perturbation bounds - the original motivation for this analysis. The proof of Proposition 1.2 is postponed to Section 4, where we also discuss some other inequalities that are classical in appearance and are suggested by our analysis. In our proof of the main result (see Proposition 2.1) we obtain some information about the spectrum of the matrices $S_{r}$. These matters are explored in more detail in Section 5, where we also raise some open questions that may be of interest.

\section{Proof of the Main Theorem}

Recall the matrix $S_{r}$ defined by (3); the $(i, i)$ entry of $S_{r}$ is to be interpreted as $p_{i}^{r-1}$. It is an important fact in the theory of operator monotone functions that $S_{r}$ is positive iff $0 \leq r \leq 1$; see [4], Chapter $\mathrm{V}$. The following proposition, needed for our proof, is therefore of independent interest.

Proposition 2.1. When $1<r<2$, the $n \times n$ matrix $S_{r}$ has just one positive eigenvalue. 
Proof. Let $J_{n}$ denote the $n \times n$ matrix whose entries are all 1 . For $1<r<2$ we have matrix convexity of the map $A \mapsto A^{r}$ (see [4], Chapter $V$ and Exercise V.2.11 in particular). Thus, for $0<t<1$ and any $K>0$ (we'll later let $K$ tend to infinity)

$$
\left((1-t) A+t K J_{n}\right)^{r} \leq(1-t) A^{r}+t K^{r} J_{n}^{r},
$$

that is,

$$
\frac{\left(A+t\left(K J_{n}-A\right)\right)^{r}-A^{r}}{t} \leq K^{r} J_{n}^{r}-A^{r} .
$$

Letting $t$ decrease towards 0 , we conclude that

$$
D A^{r}\left(K J_{n}-A\right) \leq K^{r} J_{n}^{r}-A^{r},
$$

that is,

$$
r S_{r} \circ\left(K J_{n}-A\right) \leq K^{r} J_{n}^{r}-A^{r},
$$

that is (recalling that $A$ is diagonal in this discussion)

$$
r K S_{r}-r A^{r} \leq K^{r} J_{n}^{r}-A^{r},
$$

that is,

$$
S_{r} \leq K^{r-1} \frac{1}{r} J_{n}^{r}+\frac{1}{K}\left(1-\frac{1}{r}\right) A^{r} .
$$

Letting $\lambda_{2}$ denote the second largest eigenvalue, we have by Weyl monotonicity (see [4], Corollary III.2.3)

$$
\begin{aligned}
\lambda_{2}\left(S_{r}\right) & \leq \lambda_{2}\left(K^{r-1} \frac{1}{r} J_{n}^{r}+\frac{1}{K}\left(1-\frac{1}{r}\right) A^{r}\right) \\
\leq & \lambda_{2}\left(K^{r-1} \frac{1}{r} J_{n}^{r}\right)+\left\|\frac{1}{K}\left(1-\frac{1}{r}\right) A^{r}\right\| .
\end{aligned}
$$

Now $J_{n}=n P$ where $P$ is a rank-one projection, so that $J_{n}^{r}=n^{r} P$ and $\lambda_{2}\left(J_{n}^{r}\right)=$ 0 . It follows that $\lambda_{2}\left(S_{r}\right) \leq\left\|K^{-1}\left(1-r^{-1}\right) A^{r}\right\|$, and letting $K \rightarrow \infty$ we obtain $\lambda_{2}\left(S_{r}\right) \leq 0$, as desired.

Remark. The same result could be proved for any matrix

$$
S=\left[\frac{f\left(p_{i}\right)-f\left(p_{j}\right)}{p_{i}-p_{j}}\right]
$$

in place of $S_{r}$, provided that $f$ is a matrix convex function. 
Proof of Theorem 1.1. We have explained in Section 1 that to prove the theorem it suffices to display a factorization

$$
S_{r}=R C
$$

where the rows of $R$ and the columns of $C$ have norms not exceeding 1 . Let $w_{i j}$ be the entries of the matrix $S_{r}$. Since $p_{1}=1, w_{11}=1$. So, we can start by choosing $(1,0, \ldots, 0)$ as the first row of $R$ and its transpose as the first column of $C$. Then (6) takes the form

$$
S_{r}=\left[\begin{array}{cc}
1 & x^{*} \\
x & T
\end{array}\right]=\left[\begin{array}{cc}
1 & \overrightarrow{0}^{*} \\
x & R_{1}
\end{array}\right]\left[\begin{array}{cc}
1 & x^{*} \\
\overrightarrow{0} & C_{1}
\end{array}\right],
$$

where $x$ has entries $w_{21}, \ldots, w_{n 1}$ and $C_{1}, R_{1}$ are $(n-1) \times(n-1)$ matrices such that $R_{1} C_{1}=T-x x^{*}$. Let us denote the rows of $R_{1}$ by $r_{2}, \ldots, r_{n}$ and the columns of $C_{1}$ by $c_{2}, \ldots, c_{n}$. We need to arrange that

$$
\left|w_{k 1}\right|^{2}+\left\|r_{k}\right\|^{2} \leq 1 \text { and }\left|w_{1 k}\right|^{2}+\left\|c_{k}\right\|^{2} \leq 1,
$$

for $k=2, \ldots, n$. Note that $w_{k 1}=w_{1 k}$.

If we define

$$
X=\left[\begin{array}{cc}
1 & \overrightarrow{0}^{*} \\
x & -I
\end{array}\right]
$$

where $I$ denotes the $(n-1)$-dimensional identity matrix, then

$$
X S_{r} X^{*}=\left[\begin{array}{cc}
1 & \overrightarrow{0}^{*} \\
\overrightarrow{0} & T-x x *
\end{array}\right] .
$$

By Proposition 2.1 and the Sylvester Law of Inertia (see [13]), $X S_{r} X^{*}$ has exactly one positive eigenvalue. Hence the matrix $T-x x^{*}$ does not have any positive eigenvalue. In other words, the matrix $x x^{*}-T$ is positive semidefinite. Set $Y=\operatorname{diag}\left(y_{2}, \ldots, y_{n}\right)$ where

$$
y_{k}=\frac{1}{\sqrt{1-\left|w_{k 1}\right|^{2}}} .
$$

Then $Y\left(x x^{*}-T\right) Y$ is also positive semidefinite. By a well-known theorem of Schur, the Schur multiplier norm of such a matrix is equal to its maximum diagonal entry (see [17], [14], Theorem 5.5.18). The $k$ th diagonal entry $(k=2, \ldots, n)$ of $Y\left(x x^{*}-T\right) Y$ is:

$$
\left(\left|w_{k 1}\right|^{2}-p_{k}^{r-1}\right) y_{k}^{2}
$$


We claim that none of the quantities (10) is greater than 1 . Recalling that

$$
w_{k 1}=\frac{1-p_{k}^{r}}{r\left(1-p_{k}\right)}
$$

and (9), we compute that this claim amounts to:

$$
\left(\frac{1-p_{k}^{r}}{r\left(1-p_{k}\right)}\right)^{2} \leq \frac{1+p_{k}^{r-1}}{2} .
$$

Since we have $r \geq \sqrt{2}$ by hypothesis, this claim is valid because of Proposition 1.2 .

Thus $\left\|Y\left(x x^{*}-T\right) Y\right\|_{S} \leq 1$ so that there must exist a Haagerup factorization

$$
Y\left(x x^{*}-T\right) Y=R_{2} C_{2},
$$

where the rows of $R_{2}$ and the columns of $C_{2}$ have norms not exceeding 1. This implies that the rows $r_{k}$ of the matrix $R_{1}$ defined by $R_{1}=-Y^{-1} R_{2}$ and the columns $c_{k}$ of $C_{1}=C_{2} Y^{-1}$ have norms satisfying (8). Since (12) implies that $R_{1} C_{1}=T-x x^{*}$, we have the required Haagerup factorization for $S_{r}$.

Remark. We thank the referee for the pains taken in reviewing our work. In particular, the referee has pointed out that the arguments above are close to those in the paper [11] by Cowen et al. Indeed, our result could be based directly on our Propositions 1.2 and 2.1, along with Theorem 9 from [11]. We have preferred to retain the proof above in order to make our exposition more self-contained.

It is perhaps worthwhile to point out that in the $2 \times 2$ case we could simplify the proof above, but only slightly so. We still need Haagerup's theorem and Proposition 1.2. Let us indicate this briefly.

Let $1<r<2$, and let $A=\operatorname{diag}(1, p)$, where $0<p<1$. We have

$$
S_{r}=\left[\begin{array}{cc}
1 & x \\
x & p^{r-1}
\end{array}\right], \quad x=\frac{1-p^{r}}{r(1-p)} .
$$

We want to characterize those $r$ for which $\left\|S_{r}\right\|_{S}=1$. By Haagerup's theorem this will be so iff there is a factorization

$$
\left[\begin{array}{cc}
1 & x \\
x & p^{r-1}
\end{array}\right]=\left[\begin{array}{ll}
1 & 0 \\
x & \rho
\end{array}\right]\left[\begin{array}{ll}
1 & x \\
0 & \sigma
\end{array}\right]
$$


such that $x^{2}+|\rho|^{2} \leq 1, x^{2}+|\sigma|^{2} \leq 1$, and $x^{2}+\rho \sigma=p^{r-1}$. Note that

$$
x=\int_{0}^{1}(p+t(1-p))^{r-1} d t
$$

and that $s^{r-1}$ is a concave function of $s$ when $1<r<2$. It follows that

$$
x \geq \frac{p^{r-1}+1}{2},
$$

and by the AM-GM inequality $x \geq \sqrt{p^{r-1}}$. In order to achieve $x^{2}+\rho \sigma=p^{r-1}$ we must have $\rho \sigma \leq 0$ and may therefore take $\rho=-\sigma=\sqrt{x^{2}-p^{r-1}}$. The remaining condition $x^{2}+\rho^{2} \leq 1$ is then a form of (4).

Letting $p$ tend to 0 , we see that the condition $r \geq \sqrt{2}$ is necessary for (4) to hold. Thus the equality (2) is not always true for $2 \times 2$ matrices if $1<r<\sqrt{2}$. Bhatia and Sinha [7] showed this by constructing explicit counterexamples.

\section{Perturbation Bounds}

Knowing the value of (or even upper bounds for) the norm $\|D f(A)\|$ enables one to obtain useful perturbation bounds for the operator function $f$; see [4], Section X.3. Indeed, this is the motivation for the present work and its predecessors [2], [7], and [9].

Let $A, B$ be any two matrices. Then, for each positive integer $n$, we have

$$
A^{n}-B^{n}=\sum_{j=1}^{n} A^{n-j}(A-B) B^{j-1} .
$$

It follows that

$$
\left\|A^{n}-B^{n}\right\| \leq n M^{n-1}\|A-B\|,
$$

where $M=\max (\|A\|,\|B\|)$. For positive matrices $A, B$, the theorem below gives a generalization of (16) in which integer powers are replaced by arbitrary real powers $r$ with $r \geq \sqrt{2}$.

Theorem 3.1. Let $A, B$ be positive matrices. Then for every real $r \geq \sqrt{2}$, we have

$$
\left\|A^{r}-B^{r}\right\| \leq r M^{r-1}\|A-B\|,
$$

where $M=\max (\|A\|,\|B\|)$. 
Proof. Let $A(t)=(1-t) A+t B,(0 \leq t \leq 1)$. Use the Mean Value Theorem (as in [4], Theorem X.4.5) with $f(A)=A^{r}$, and the chain rule to get

$$
\begin{aligned}
\left\|A^{r}-B^{r}\right\| & \leq \sup _{0 \leq t \leq 1}\|D f(A(t))\| \cdot\left\|A^{\prime}(t)\right\| \\
& =\sup _{0 \leq t \leq 1}\|D f(A(t))\| \cdot\|A-B\| .
\end{aligned}
$$

If $r \geq \sqrt{2}$, then by Theorem 1.1 (for $r \in[\sqrt{2}, 2)$ ) and Corollary 4 of [7] (for $r \geq 2$ )

$$
\|D f(A(t))\|=r\left\|(A(t))^{r-1}\right\| \leq r M^{r-1} .
$$

Combining (18) and (19), one gets (17).

We have obtained (17) as a consequence of (19), which is not valid for $1<$ $r<\sqrt{2}$. One may wonder whether (17) follows by some other argument for these exceptional values. The answer is no - the inequality (17) for $r>1$ implies the corresponding sharp estimate for $\left\|D A^{r}\right\|$. To see this note that if (17) holds, then we have, for all positive $A$ and small Hermitian $H$,

$$
\begin{aligned}
\left\|(A+H)^{r}-A^{r}\right\| \leq r(\|A\|+\|H\|)^{r-1}\|H\| \\
=r\|A\|^{r-1}\left(1+\frac{\|H\|}{\|A\|}\right)^{r-1}\|H\| \\
=r\|A\|^{r-1}\left(1+\sum_{n=1}^{\infty} \frac{(r-1)(r-2) \ldots(r-n)}{n !}\left(\frac{\|H\|}{\|A\|}\right)^{n}\right) \cdot\|H\| \\
=r\|A\|^{r-1}\|H\|+O\left(\|H\|^{2}\right) .
\end{aligned}
$$

Hence $\left\|D A^{r}(H)\right\| \leq r\left\|A^{r-1}\right\| \cdot\|H\|$ and this implies $\left\|D A^{r}\right\|=\left\|r A^{r-1}\right\|$, as claimed.

There are, however, perturbation bounds of a somewhat different character that do apply for $r \leq \sqrt{2}$; the following proposition, for example, applies when $1 \leq r \leq 2$.

Proposition 3.2. Suppose that $0<m I \leq A, B \leq M I$, and that $1 \leq r \leq 2$. Then

$$
\left\|A^{r}-B^{r}\right\| \leq M\left(M^{r-2}+(r-1) m^{r-2}\right)\|A-B\| .
$$


Remark. For $r=1$ and $r=2$ this inequality is sharp.

Proof. First observe that

$$
\begin{aligned}
\left\|A^{r}-B^{r}\right\| & =\left\|A^{r-1}(A-B)+\left(A^{r-1}-B^{r-1}\right) B\right\| \\
& \leq\|A\|^{r-1}\|A-B\|+\left\|A^{r-1}-B^{r-1}\right\| \cdot\|B\| .
\end{aligned}
$$

If, moreover, $A, B \geq m I>0$, then

$$
\left\|A^{r-1}-B^{r-1}\right\| \leq(r-1) m^{r-2}\|A-B\|
$$

follows from [2] (see [4], p. 305). Combining the last two inequalities, we obtain (20).

In place of (15), we may try to exploit the identity

$$
A^{n}-B^{n}=\frac{1}{n} \sum_{k=0}^{n-1} \prod_{j=0}^{n-1}\left(A-\omega^{j+k} B\right),
$$

where $\omega=e^{2 \pi i / n}$. By a theorem of Bhatia and Kittaneh (see [5]),

$$
A, B \geq 0 \Rightarrow\left\|A-\omega^{j+k} B\right\| \leq\|A+B\| .
$$

Hence, for $A, B \geq 0$ and $n=1,2, \ldots$,

$$
\left\|A^{n}-B^{n}\right\| \leq\|A+B\|^{n-1}\|A-B\| .
$$

When $B$ is a small perturbation of $A,\|A+B\| \approx 2 M$ and (22) is much weaker than (16). In other situations (22) may be better, and we may ask whether there is a generalization replacing $n$ by real $r \geq 1$. That this is not the case follows from the connections we have drawn between such inequalities and the Fréchet derivative. If we had

$$
\left\|A^{r}-B^{r}\right\| \leq\|A+B\|^{r-1}\|A-B\|,
$$

then for $A \approx B$

$$
\left\|A^{r}-B^{r}\right\| \lesssim 2^{r-1} M^{r-1}\|A-B\|,
$$

so that

$$
\left\|D A^{r}\right\| \leq 2^{r-1}\left\|A^{r-1}\right\| .
$$

Now for $r \in(1,2), 2^{r-1}<r$, so this is impossible. 


\section{Classical inequalities}

The result of Proposition 1.2,

$$
0<p<1, r \geq \sqrt{2} \Rightarrow\left(\frac{1-p^{r}}{r(1-p)}\right)^{2} \leq \frac{1+p^{r-1}}{2},
$$

is classical in appearance. We have remarked, however, that our proof seems to require unusual techniques.

Proof of Proposition 1.2. We first remark that when $r \in\left[\frac{3}{2}, 2\right]$ this result can be based rather directly on the concavity of $s^{r-1}$ and convexity of $s^{2(r-1)}$. Indeed, using the representation (14), another application of the concavity of the integrand yields

$$
\frac{1-p^{r}}{r(1-p)} \leq\left(\frac{1+p}{2}\right)^{r-1}
$$

The convexity of $s^{2(r-1)}$ then implies that

$$
\left(\frac{1-p^{r}}{r(1-p)}\right)^{2} \leq\left(\frac{1+p}{2}\right)^{2(r-1)} \leq \frac{1+p^{2(r-1)}}{2} .
$$

Since $p \leq 1$, (24) implies (4). It is also clear that (4) holds for $r \geq 2$, since $s^{r-1}$ is then convex and (14) shows that, when $r \geq 2$

$$
\left(x^{2} \leq\right) \quad x \leq \frac{1+p^{r-1}}{2} .
$$

For the case $r \in\left[\sqrt{2}, \frac{3}{2}\right)$, however, we have not found a replacement for the rather intricate and unusual argument that follows.

Let $p$ tend to 0 to see that $r \geq \sqrt{2}$ is necessary. To see that it is also sufficient we approximate $r$ in $[\sqrt{2}, 2)$ by rationals $m / n$ with $m<2 n$ and $m^{2}>2 n^{2}$.

Let $u=p^{1 / n}$ so that, with $r=m / n$, (4) becomes

$$
2 n^{2}\left(1-u^{m}\right)^{2} \leq m^{2}\left(1+u^{m-n}\right)\left(1-u^{n}\right)^{2} .
$$

Cancelling $(1-u)^{2}$ from both sides we have

$$
2 n^{2}\left(1+u+\cdots+u^{m-1}\right)^{2} \leq m^{2}\left(1+u^{m-n}\right)\left(1+u+\cdots+u^{n-1}\right)^{2} .
$$

For each $k=0,1,2, \ldots$, let $L(k)$ be the coefficient of $u^{k}$ on the LHS of (26), and let $R(k)$ be the corresponding coefficient on the RHS. The coefficients $L(k)$ and $R(k)$ are integers and our argument is based on the sign pattern of $R(k)-L(k)$ as 
$k$ increases. Note that $\left(1+u+\cdots+u^{m-1}\right)^{2}=1+2 u+3 u^{2}+\cdots+m u^{m-1}+$ $(m-1) u^{m}+\cdots+2 u^{2 m-3}+u^{2(m-1)}$, so that $L(k)$ has a triangular graph with slope $+2 n^{2}$ from $k=0$ to $k=m-1$ and slope $-2 n^{2}$ from $k=m-1$ to $k=2(m-1)$; for $k>2(m-1), L(k)=0$. Similarly, the graph of $R(k)$ is the superposition of two triangular graphs corresponding to

$$
\begin{gathered}
\left(1+u+\cdots+u^{n-1}\right)^{2}, \\
u^{m-n}\left(1+u+\cdots+u^{n-1}\right)^{2} .
\end{gathered}
$$

In following our argument the reader may find it helpful to sketch and compare the graphs of $L(k)$ and $R(k)$. Since the first factor of (28) shifts the triangular graph by $m-n<n$ units to the right, the superposition of (27) and (28) yields a graph with slope $+1(k=0$ to $m-n-1)$, slope $+2(k=m-n-1$ to $n-1)$, slope $0(k=n-1$ to $(n-1)+(m-n)=m-1)$, slope $-2(k=m-1$ to $2(n-1)+1=2 n-1)$, and slope $-1(k=2 n-1$ to $n+m-2)$. For $k>n+m-2$, $R(k)=0$.

Now $m^{2}>2 n^{2}$, so that $R(0)-L(0)>0$, and because the slope of $R(\cdot)$ is $m^{2}$ or $2 m^{2}$ in the range $k=0$ to $n-1, R(k)-L(k)$ remains positive there. At some $k^{*} \geq n, R(k)-L(k)$ becomes negative. We claim it then remains negative until $k>2 m-2$, where both $R(k)$ and $L(k)$ are 0 . To see this note that for $k \geq n$ there are three possibilities:

(i) the slope of $R(\cdot)$ is 0 while that of $L(\cdot)$ is $+2 n^{2}$ (between $k=n-1$ and $k=m-1)$;

(ii) the slope of $R(\cdot)$ is $-2 m^{2}$ or $-m^{2}$ while that of $L(\cdot)$ is $-2 n^{2}$ (between $k=m-1$ and $k=n+m-2)$;

(iii) $R(k)=0$ while $L(k)>0$ (between $k=n+m-1$ and $k=2(m-1)$ ).

In cases (i) and (ii), $R(k)-L(k)$ remains negative because $R(\cdot)$ is decreasing faster than $L(\cdot)$, whereas in case (iii) $R(k)-L(k)$ is clearly negative. We remark that case (i) is not actually needed since it may be shown that $R(m-1)>L(m-1)$ (exercise), that is, $k^{*} \geq m$.

Finally, we note that (26) is equivalent to

$$
\sum_{k=0}^{2(m-1)}(R(k)-L(k)) u^{k} \geq 0 .
$$

In view of the foregoing argument, we may express this as

$$
\sum_{k=0}^{k^{*}-1} C(k) u^{k}-\sum_{k=k^{*}}^{2(m-1)} C(k) u^{k} \geq 0,
$$


where each $C(k) \geq 0$. Now $0<u<1$ so that $u^{k}$ decreases with $k$ yet is positive. It follows that

$$
\sum_{k=0}^{k^{*}-1} C(k) u^{k} \geq\left(\sum_{k=0}^{k^{*}-1} C(k)\right) u^{k^{*}}
$$

and

$$
\sum_{k=k^{*}}^{2(m-1)} C(k) u^{k} \leq\left(\sum_{k=k^{*}}^{2(m-1)} C(k)\right) u^{k^{*}}
$$

Hence it is sufficient to show that

$$
\sum_{k=0}^{k^{*}-1} C(k) \geq \sum_{k=k^{*}}^{2(m-1)} C(k)
$$

In fact we have equality in (33):

$$
\sum_{k=0}^{k^{*}-1} C(k)-\sum_{k=k^{*}}^{2(m-1)} C(k)=\sum_{k=0}^{2(m-1)}(R(k)-L(k)) 1^{k}=0,
$$

because each side of (26) equals $2 n^{2} m^{2}$ when $u=1$.

Certain other inequalities of classical type are hidden in the results of Section 2. Perhaps a more direct proof can be found, for example, for the inequality of the following proposition.

Proposition 4.1. Suppose that $1<r<2$. Given $0<q<p$, let

$$
w(p, q)=\frac{p^{r}-q^{r}}{r(p-q)}
$$

Then

$$
\left(w^{2}(1, p)-p^{r-1}\right)\left(w^{2}(1, q)-q^{r-1}\right) \geq(w(1, p) w(1, q)-w(p, q))^{2}
$$


Remark. For some rational values of $r$ (eg $r=3 / 2$ ), the inequality (34) is equivalent to the nonnegativity of reasonably simple polynomials in $p$ and $q$. In these cases it may be feasible to prove (34) algebraically, especially if one uses a symbolic manipulator such as MAPLE or Mathematica.

Proof. Apply Proposition 2.1 to the case where $n=3$ and $A$ has eigenvalues $1, p, q$. Since $S_{r}$ has just one positive eigenvalue, we can argue as in the proof of Theorem 1.1 that $x x^{*}-T$ is positive semidefinite; but in this situation

$$
x x^{*}-T=\left[\begin{array}{cc}
w^{2}(1, p)-p^{r-1} & w(1, p) w(1, q)-w(p, q) \\
w(1, p) w(1, q)-w(p, q) & w^{2}(1, q)-q^{r-1}
\end{array}\right] .
$$

Thus (34) reflects the fact that $\operatorname{det}\left(x x^{*}-T\right) \geq 0$.

It is a curious fact that the nonnegative definite matrices $x x^{*}-T$ obtained as in (35) are nonnegative also in the entrywise sense. This is the case no matter what is the size $n$ of the matrix $A$, as the following proposition (with its classical proof) shows.

Proposition 4.2. Let $w(p, q)$ be as in Proposition 4.1. Then:

$$
w(1, p) w(1, q) \geq w(p, q) .
$$

Proof. We shall show that, for $q<p<t<1$,

$$
w(1, p) w(t, q) \geq w(p, q) w(1, t) .
$$

Then (36) will follow by letting $t$ tend to 1 . We need to show that the ratio $R(t)=w(t, q) / w(1, t)$ is nondecreasing over the interval $[p, 1)$; we shall check that $R^{\prime}(t) \geq 0$, which is equivalent to

$$
w^{\prime}(t, q) w(1, t) \geq w^{\prime}(1, t) w(t, q),
$$

where the primes indicate differentiation with respect to $t$. Routine computations show that

$$
w^{\prime}(t, q)=\frac{t^{r-1}-w(t, q)}{t-q}
$$

so that (38) says

$$
t^{r-1}\left(\frac{w(1, t)}{t-q}+\frac{w(t, q)}{1-t}\right) \geq w(t, q) w(1, t)\left(\frac{1}{t-q}+\frac{1}{1-t}\right),
$$


that is,

$$
t^{r-1}((1-t) w(1, t)+(t-q) w(t, q)) \geq w(t, q) w(1, t)(1-q)
$$

that is,

$$
t^{r-1} w(1, q) \geq w(t, q) w(1, t)
$$

Setting $t_{+}(s)=t+s(1-t)$ and $t_{-}(s)=t-s(t-q)$, we have

$$
t=\frac{1-t}{1-q} t_{-}(s)+\frac{t-q}{1-q} t_{+}(s),
$$

so that the concavity of $s^{r-1}$ implies that

$$
t^{r-1} \geq \frac{1-t}{1-q} t_{-}^{r-1}(s)+\frac{t-q}{1-q} t_{+}^{r-1}(s) .
$$

Integrating over $[0,1]$ with respect to $s$ we obtain

$$
t^{r-1} \geq \frac{1-t}{1-q} w(t, q)+\frac{t-q}{1-q} w(1, t)
$$

so that the (weighted) AGM inequality yields

$$
t^{r-1} \geq(w(t, q))^{(1-t) /(1-q)}(w(1, t))^{(t-q) /(1-q)} .
$$

Since

$$
w(1, q)=\frac{t-q}{1-q} w(t, q)+\frac{1-t}{1-q} w(1, t),
$$

the AGM inequality also yields

$$
w(1, q) \geq(w(t, q))^{(t-q) /(1-q)}(w(1, t))^{(1-t) /(1-q)} .
$$

Multiplying the inequalities (40) and (41), we obtain (39). 


\section{THE SPECTRUM OF $S_{r}$ FOR LARGER $r$}

It is known that $S_{r}$ is (strictly) positive definite if $r \in(0,1)$ and the $p_{k}$ are distinct; on the other hand, Proposition 2.1 shows that $S_{r}$ has just one positive eigenvalue if $r \in(1,2)$. Here we consider the eigenvalue variation of $S_{r}$ in a broader context. Since we are concerned mainly with the signs of eigenvalues here, we ignore the scalar $1 / r$ and replace $S_{r}$ with the $n \times n$ matrix

$$
Q_{r}=\left[\frac{p_{i}^{r}-p_{j}^{r}}{p_{i}-p_{j}}\right]
$$

Here it is understood that $r>0$, that the $p_{k}$ are distinct positive reals, and that the $k$-th diagonal entry of $Q_{r}$ is interpreted as the derivatve $r p_{k}^{r-1}$.

In Figure 1 we see the typical variation of the eigenvalue signs of $Q_{r}$ as $r$ increases. The fact that $Q_{r}$ is strictly positive definite when $r \in(0,1)$ is a reflection of the well-known operator monotonicity of $t^{r}$ in that range (see [4], Chapter V, for example). One efficient and direct way to see whether $Q_{r}>0$ is to note that

$$
Q_{r}=A^{(r-1) / 2}\left[\varphi_{r}\left(\frac{t_{i}-t_{j}}{2}\right)\right] A^{(r-1) / 2}
$$

where $A=\operatorname{diag}\left(p_{1}, \ldots, p_{n}\right), p_{k}=e^{t_{k}}$, and $\varphi_{r}(t)=\sinh (r t) / \sinh (t)$. Thus $Q_{r}>0$ iff $\varphi_{r}$ is (strictly) positive definite; for a fuller range of the applications of this idea, see Bhatia and Parthasarathy [6]. For $r \in(0,1), \varphi_{r}$ is the Fourier transform of

$$
\psi_{r}(s)=\frac{\sin r \pi}{\cosh s \pi+\cos r \pi}
$$

(we ignore inessential constants in taking Fourier transforms). Since $\psi_{r}(s)$ is strictly positive (for $r \in(0,1)$ ), it follows that $Q_{r}>0$. Certain features of Figure 1 are now clear: all eigenvalues are positive over the range $0<r<1$ and, by Proposition 2.1, just one is positive over the range $1<r<2$. At $r=1$, of course, we see eigenvalues reflecting the fact that $Q_{1}$ is $n$ times a rank-one projection. The following proposition explains some additional features of Figure 1. We denote the inertia vector of a Hermitian matrix $T$ by $\operatorname{In}(T)$; that is,

$$
\operatorname{In}(T)=(\pi(T), \zeta(T), v(T)),
$$

where $\pi(T)$ is the number of positive eigenvalues of $T, \zeta(T)$ is the number of zero eigenvalues of $T$, and $v(T)$ is the number of negative eigenvalues of $T$. 


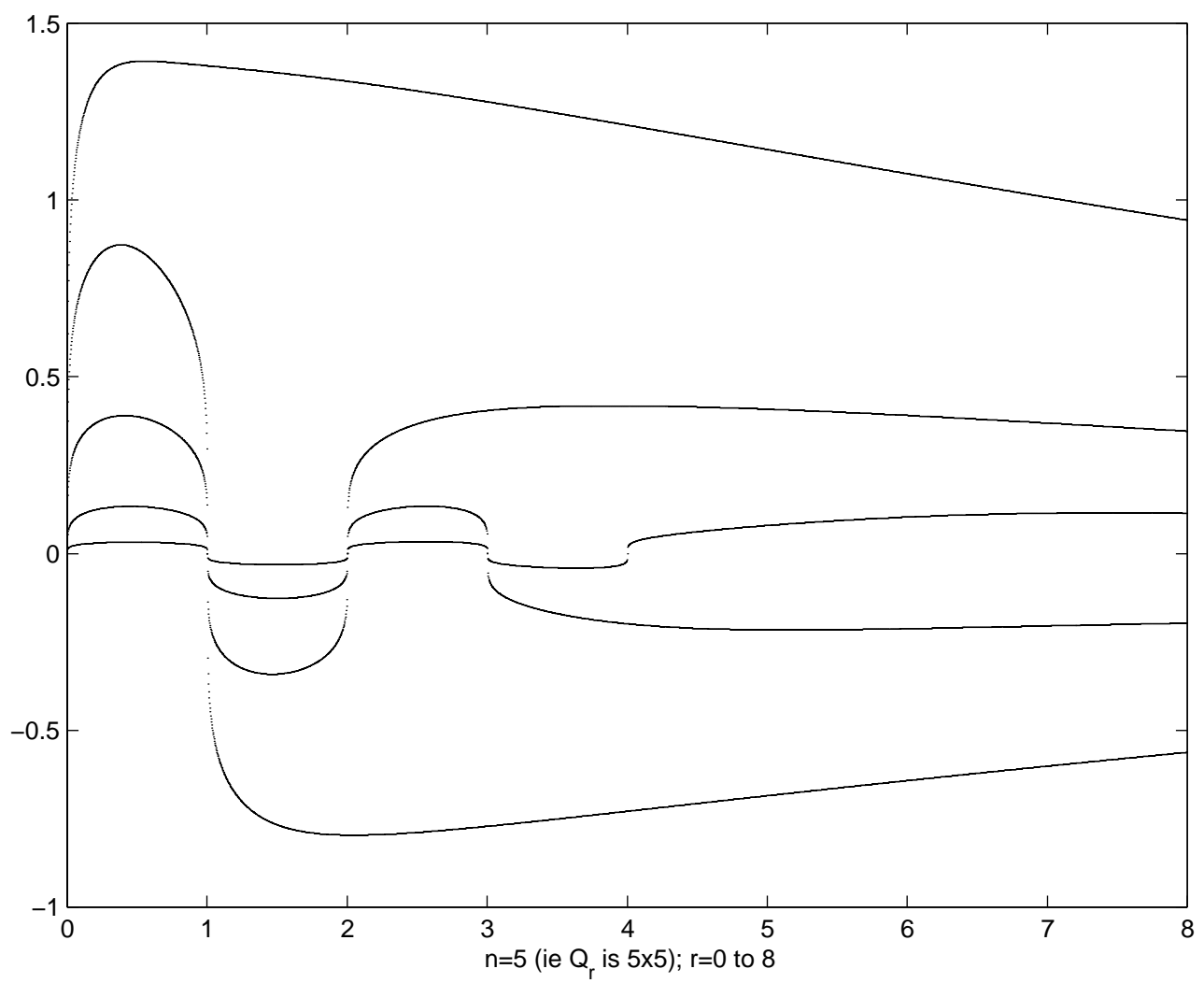

FIGURE 1. Here we see a typical pattern of sign changes in the eigenvalues of $S_{r}$. In this example the $A=$ $\operatorname{diag}\left(p_{1}, p_{2}, p_{3}, p_{4}, p_{5}\right)$ where the $p_{k}$ have been chosen at random from $(0,1)$. Thus $n=5$ and we have displayed the five eigenvalues (suitably scaled) of $S_{r}$ as $r$ ranges over $(0,8)$. Since some eigenvalues tend to be very close to 0 , the vertical scale in Figure 1 is greatly expanded near 0 , so that the eigenvalue signs are easily followed. The figure was produced with the help of MATLAB software.

Proposition 5.1. If $r$ is a positive integer not exceeding $n$, then

$$
r=2 k \Rightarrow \operatorname{In}\left(Q_{r}\right)=(k, n-r, k),
$$

and

$$
r=2 k-1 \Rightarrow \operatorname{In}\left(Q_{r}\right)=(k, n-r, k-1) .
$$


If $r$ is an integer greater than $n$, then $\operatorname{In}\left(Q_{r}\right)=\operatorname{In}\left(Q_{n}\right)$.

Proof. Let $V$ be the $r \times r$ order-reversing permutation matrix (1's along the anti-diagonal) and let $R$ be the $r \times r$ positive (Gram) matrix

$$
R=\left[\left(\underline{p}^{i-1}, \underline{p}^{j-1}\right)\right],
$$

where

$$
\underline{p}^{k}=\left[\begin{array}{c}
p_{1}^{k} \\
\vdots \\
p_{n}^{k}
\end{array}\right]
$$

Note that $R$ may be computed as

$$
\left[c_{i+j-2}\right], \quad \text { where } c_{j}=\sum_{k=1}^{n} p_{k}^{j} .
$$

Writing

$$
\frac{p_{i}^{r}-p_{j}^{r}}{p_{i}-p_{j}}=p_{i}^{r-1}+p_{i}^{r-2} p_{j}+\cdots+p_{j}^{r-1}
$$

we see that

$$
Q_{r}=\underline{p}^{r-1} \cdot\left(\underline{p}^{0}\right)^{*}+\underline{p}^{r-2} \cdot\left(\underline{p}^{1}\right)^{*}+\cdots+\underline{p}^{0} \cdot\left(\underline{p}^{r-1}\right)^{*},
$$

so that

$$
Q_{r} \underline{p}^{k}=\sum_{j=0}^{r-1}\left(\underline{p}^{k}, \underline{p}^{r-1-j}\right) \underline{p}^{j} .
$$

Let $L=\operatorname{span}\left\{\underline{p}^{0}, \ldots, \underline{p}^{r-1}\right\}$. It is clear from (49) that $Q_{r} \equiv 0$ on $L^{\perp}$ and that $L$ is invariant under $\overline{Q_{r}}$. Since we are assuming that the $p_{k}$ are distinct, the vectors $\underline{p}^{j}$ are linearly independent. With respect to the basis $\underline{p}^{0}, \ldots, \underline{p}^{r-1}$, the restriction of $Q_{r}$ to $L$ has the matrix $M$, where $m_{i j}=\left(\underline{p}^{i-1}, \underline{p}^{r-j}\right)$ by (49). Hence $M=\left[c_{r+i-j-1}\right]$, using the notation of (47). In terms of the matrices $V$ and $R$ introduced above, $M=R V$. Thus the spectrum of $Q_{r}$ consists of $n-r 0$ 's from $L^{\perp}$ along with the eigenvalues of $R V$ (which are nonzero).

Since $R V$ is similar to $R^{1 / 2} V R^{1 / 2}$ we may examine the eigenvalues of the latter. By Sylvester's law of inertia, their signs follow the same sign pattern as those of $V$. Now $V$ is unitary and Hermitian, so that its eigenvalues are \pm 1 . The trace 
of $V$ is clearly 1 if $r$ is odd and 0 if $r$ is even. Hence $V$ has eigenvalue -1 with multiplicity $[r / 2]$ and has eigenvalue +1 with multiplicity $r-[r / 2]$.

Remark. Although our focus has been on positive values of $r$, the relation $Q_{-r}=A^{-r}\left(-Q_{r}\right) A^{-r}$ (and Sylvester's law of inertia) allow us to transfer our results on eigenvalue sign patterns to negative values of $r$ as well.

The results of this section lead us to make the following conjecture.

Conjecture. If $0<r<n$ and $r$ is not an integer, then

$$
[r]=2 k \Rightarrow \operatorname{In}\left(Q_{r}\right)=(n-k, 0, k),
$$

and

$$
[r]=2 k-1 \Rightarrow \operatorname{In}\left(Q_{r}\right)=(k, 0, n-k) .
$$

If $r>n$, then $\operatorname{In}\left(Q_{r}\right)=\operatorname{In}\left(Q_{n}\right)$.

\section{ACKNOWLEDGEMENTS}

We are most grateful to the University of Guelph sabbatical program, to NSERC of Canada, to the Indian Statistical Institute, and to the Maui High Performance Computing Center, for their support of this work. Thanks are also due to K. B. Sinha, for many helpful discussions of the problems treated here. Some of the techniques used in Section 2 were suggested by a fortunate discussion of multivariate Pick interpolation with F. H. Szafraniec, who directed us to Quiggin's paper [16] and to [18].

\section{REFERENCES}

[1] J. R. Angelos, C. C. Cowen, And S. K. Narayan, Triangular truncation and finding the norm of a Hadamard multiplier, Linear Alg. Appl. 170 (1992), 117136.

[2] R. BHATIA, First and second order perturbation bounds for the operator absolute value, Linear Alg. Appl. 208 (1994), 367-376.

[3] R. BhatiA, Perturbation bounds for the operator absolute value, Linear Alg. Appl. 226 (1995), 639-645.

[4] R. Bhatia, Matrix Analysis, Springer Verlag, New York, 1997.

[5] R. BHATIA AND F. KITTANEH, Norm inequalities for positive operators, Letters in Mathematical Physics 43 (1998), 225-231.

[6] R. Bhatia And K. R. Parthasarathy, Positive definite functions and operator inequalities, Bull. London Math. Soc. 32 (2000), 214-228. 
[7] R. BHATIA AND K. B. SINHA, Variation of real powers of positive operators, Indiana Univ. Math. J. 43 (1994), 913-925.

[8] R. Bhatia And K. B. Sinha, Derivations, derivatives, and chain rules, Linear Alg. Appl. 302/303 (1999), 231-244.

[9] R. Bhatia, D. Singh, AND K. B. SinHA, Differentiation of operator functions and perturbation bounds, Commun. Math. Phys. 191 (1998), 603-611.

[10] C. C. Cowen, M. A. DritsChel, AND R. C. Penney, Norms of Hadamard multipliers, SIAM J. Matrix Anal. Appl. 15 (1994), 313-320.

[11] C. COWEn, K. DeBro, AND P. SEPANSKI, Geometry and the norms of Hadamard multipliers, Linear Alg. Appl. 218 (1995), 234-249.

[12] U. HaAgerup, Decompositions of completely bounded maps on operator algebras, preprint 1984.

[13] R. A. Horn And C. R. Johnson, Matrix Analysis, Cambridge University Press, 1985.

[14] R. A. Horn and C. R. Johnson, Topics in Matrix Analysis, Cambridge University Press, 1991.

[15] R. Mathias, Matrix completions, norms, and Hadamard products, Proc. Amer. Math. Soc. 117 (1993), 905-918.

[16] P. QUIGGIN, For which reproducing kernel Hilbert spaces is Pick's theorem true?, Integr. Equat. Oper. Th. 16 (1993), 244-266.

[17] I. SCHUR, Bemerkungen zur Theorie der beschränkten Bilinearformen mit unendlich vielen Veränderlichen, J. Reine Angew. Math. 140 (1911), 1-28.

[18] F. H. SZAFRANIEC, On bounded holomorphic interpolation in several variables, Monatsh. Math. 101 (1986), 59-66.

RAJENDRA BHATIA

Indian Statistical Institute

7, S. J. S. Sansanwal Marg

New Delhi-110016, INDIA

EMAIL: rbh@isid1.isid.ac.in

JoHn A. Holbrook

Mathematics and Statistics

University of Guelph

Guelph, Ontario N1G 2W1, CANADA

EMAIL: jholbroo@math.uoguelph.ca

Subject Classification: Primary 47A55; Secondary 58C20

Submitted: February 19th, 1999, revised: September 20th, 1999. 